\title{
Do we care about the powerless third? An ERP study of the three-person ultimatum game
}

\author{
Johanna Alexopoulos ${ }^{1,2}{ }^{*}$, Daniela M. Pfabigan ${ }^{1}$, Claus Lamm ${ }^{1}$, Herbert Bauer ${ }^{1}$ and \\ Florian Ph. S. Fischmeister ${ }^{1,3}$
}

${ }^{1}$ Faculty of Psychology, Social, Cognitive and Affective Neuroscience Unit, University of Vienna, Vienna, Austria

2 Department of Psychoanalysis and Psychotherapy, Medical University Vienna, Vienna, Austria

${ }^{3}$ Department of Neurology, Study Group Clinical fMRI, Medical University of Vienna, Austria

Edited by:

Leonhard Schilbach, Max-Planck-Institute for Neurological Research, Germany

Reviewed by:

Lutz Jäncke, University of Zurich, Switzerland

Bernd Weber, Rheinische-

Friedrich-Wilhelms Universität,

Germany

\section{*Correspondence:}

Johanna Alexopoulos, Faculty of Psychology, Social, Cognitive and Affective Neuroscience Unit,

University of Vienna, Liebiggasse 5 ,

Wien, 1010, Austria.

e-mail: johanna.alexopoulos@

univie.ac.at
Recent years have provided increasing insights into the factors affecting economic decision-making. Little is known about how these factors influence decisions that also bear consequences for other people. We examined whether decisions that also affected a third, passive player modulate the behavioral and neural responses to monetary offers in a modified version of the three-person ultimatum game. We aimed to elucidate to what extent social preferences affect early neuronal processing when subjects were evaluating offers that were fair or unfair to themselves, to the third player, or to both. As an event-related potential (ERP) index for early evaluation processes in economic decision-making, we recorded the medial frontal negativity (MFN) component in response to such offers. Unfair offers were rejected more often than equitable ones, in particular when negatively affecting the subject. While the MFN amplitude was higher following unfair as compared to fair offers to the subject, MFN amplitude was not modulated by the shares assigned to the third, passive player. Furthermore, rejection rates and MFN amplitudes following fair offers were positively correlated, as subjects showing lower MFN amplitudes following fair offers tended to reject unfair offers more often-but only if those offers negatively affected their own payoff. Altogether, the rejection behavior suggests that humans mainly care about a powerless third when they are confronted with inequality as well. The correlation between rejection rates and the MFN amplitude supports the notion that this ERP component is also modulated by positive events and highlights how our expectations concerning other humans' behavior guide our own decisions. However, social preferences like inequality aversion and concern for the well-being of others are not reflected in this early neuronal response, but seem to result from later, deliberate and higher-order cognitive processes.

Keywords: MFN, ultimatum game, social preferences, altruism, egoism, inequality aversion

\section{INTRODUCTION}

Most economic models assume that people are solely motivated by their own material payoff, i.e., they always choose what is best for them. In recent years, this view, the so called self-interest hypothesis, has been questioned. A well-known experiment which shows that this hypothesis does not fully account for human behavior in economic decision-making is the so called ultimatum game (Güth et al., 1982). In this two-person game a proposer has to split a certain amount of money between a responder and himself followed by the decision of the responder whether to accept or reject it. If the offer is accepted the money is allotted accordinglyhowever, if the responder rejects the offer both players receive nothing. Assuming that both players behave rationally and thus do not care about the outcome of the other, the responder would have to accept any positive outcome and the proposer should offer the smallest amount of money. In reality, most of the offers accepted by the responder are about $40-50 \%$ of the total amount while offers below $20 \%$ are rejected with a probability of about 50\% (Güth et al., 1982; Camerer and Richard, 1995).
Observations like these which indicate that people are selfinterested but also inequality averse led to a reformulation of models of economic decision-making, and the addition of "otherregarding" preferences to these models. More specifically, models of social preferences assume that people compare their own material payoff either with the payoff of each other player (Fehr and Schmidt, 1999) or with the average payoff across all players involved (Bolton and Ockenfels, 2000). People feel inequity if they are worse off or better off than their reference players, leading to a reduction of utility. This reduction is larger for disadvantageous inequality, i.e., being worse off than others in material terms, than for advantageous inequality, i.e., being better off (Fehr and Schmidt, 1999).

Recent event-related potential (ERP) studies document that the distinction between equitable and disadvantageous inequitable offers is already reflected by differences in an ERP component occurring $270 \mathrm{~ms}$ after the onset of an offer in the ultimatum game (Polezzi et al., 2008). Characterized by a negative deflection and being more pronounced with regard to inequitable 
as compared to equitable offers, this component was interpreted in terms of the medial frontal negativity (MFN; Gehring and Willoughby, 2002). These authors were one of the first who observed such a negative deflection in the ERP after monetary losses compared to gains. Dipole source modeling and results from studies using fMRI suggest the MFN signal to be generated in the anterior cingulate cortex (ACC; Gehring and Willoughby, 2002; Martin et al., 2009). Later studies supported this finding and proposed the MFN signal to be related to the reinforcement learning system (Holroyd and Coles, 2002). The reinforcement theory states that the midbrain dopaminergic system codes the subjective value of a certain outcome or object as a function of expectancy. In that sense predicted rewards cause a phasic activation of dopaminergic neurons whereas the omission of a reward leads to a depression. As predicted rewards do not increase the firing rate, it is assumed that the mesencephalic dopaminergic system creates a reward prediction error signal which is conveyed to cortical regions (e.g., the ACC) to allow for the adaption of the behavior (Schultz, 1999, 2010).

In line with this assumption the MFN is usually observed in tasks reflecting monetary losses after the onset of negative feedback (Gehring and Willoughby, 2002), or after feedback indicating an incorrect response (Miltner et al., 1997; Holroyd and Coles, 2002). Furthermore, the amplitude of the MFN is related to subjective values like social norms (Boksem and de Cremer, 2010), i.e., being more pronounced following unfair offers compared to fair offers when subjects are highly concerned by social norms. These findings among others led to the suggestion that the MFN is apparent whenever favorable or unfavorable events are evaluated along an abstract "good-bad" dimension (Nieuwenhuis et al., 2004).

Along these lines one might speculate that the MFN indicates the loss of utility when perceiving disadvantageous inequality. Proposing that humans are inequality averse, unbalanced offers unfair to one of the participants should be associated with more pronounced MFN amplitudes than symmetrical offers. This view is supported by behavioral studies showing that people prefer an equal split (Güth et al., 2007) and recent neuroimaging studies reporting ACC activity during the processing of unfair offers (Sanfey et al., 2003; Guroglu et al., 2011) Haruno and Frith (2010) (using a different experimental paradigm) also found increased activity in the ACC in relation to trials where participants received less than their counterparts. Activation was independent of individual differences in social value orientation.

The standard ultimatum game represents a useful behavioral paradigm to study social aspects of decision-making as it is simple and has been studied extensively within various disciplines and using different methods (for a review see Rilling and Sanfey, 2011). Nevertheless, this simplicity comes with some major limitations. For instance, it presupposes that players have equal needs for the payoff as it is usually played in an anonymous context. In contrast, in a natural environment people mostly know with whom they interact or have at least some information about their counterpart. Another major disadvantage of the standard ultimatum game is the fact that players decide only for themselves, and decisions are not influenced by the presence of other people or groups. However, in real life we hardly make decisions independently of others as others usually observe or are even able to affect our decisions by their mere presence. The present study therefore attempted to overcome this limitation by adding a third player to the standard ultimatum game setup.

As already mentioned, an MFN can be observed whenever subjects feel unfairly treated in the standard ultimatum game. Yet, the question remains how subjects evaluate offers when the proposer behaves unfairly toward someone else and when the decisions made affect this third person as well. In particular, we are interested in how the MFN amplitude is related to advantageous inequality as compared to disadvantageous inequality as well as to equity. In the present ERP study this question will be systematically addressed by introducing a third player to the original ultimatum game. In this version of the ultimatum game, originally developed by Güth and van Damme (1998), a given sum of money is split up between three players: the proposer, the responder, and a dummy player, reflecting the powerless third. If accepted by the subjects in the role of the responder, the money will be allocated according to the split offered by the proposer; otherwise, no player receives any money. The powerless third is in a yoke-situation and has no decision role in the game. This way, it is possible to study the relation between advantageous inequality (receiving more than the third player) and disadvantageous inequality (receiving less than the third player). Furthermore, by having such a fixed reference agent (the third dummy player) in contrast to the consistently changing proposers (Sanfey et al., 2003; Polezzi et al., 2008; Boksem and de Cremer, 2010) it is possible to focus on the impact of social motives that occur in strategic social interactions.

\section{MATERIALS AND METHODS PARTICIPANTS}

Eighteen undergraduate students (six male; mean age = $23.2 \pm 2.7$ years) from the University of Vienna participated in the experiment. Two of these subjects had to be excluded from further analysis since post-experimental debriefing revealed that they had not believed in the existence of a third player.

All subjects were naive to the experiment, had normal or adequately corrected vision, and were healthy and right-handed, as assessed by the Edinburgh Handedness Inventory (EHI; Oldfield, 1971). Scores for the EHI were above 70 in all subjects. Subjects were paid for their participation the amount of money they earned in four randomly chosen trials, resulting in earnings between 15 and 20 Euros on average. Written informed consent from each participant was obtained prior to the experiment. The study was conducted in accordance with the Declaration of Helsinki (1973, revised in 1983) and local guidelines and regulations of the University of Vienna and the Faculty of Psychology.

\section{STIMULUS MATERIAL}

In order to design the experimental setting, realistic offers were collected pre-experimentally following the strategy method introduced by Selten (1965). To this end, we created six possible allocations with a total sum of 15 Euros as well as another six with a total of 12 Euros. Students from different Universities in Vienna were asked to choose one offer from each group resulting in two different offers for each student. After they had chosen the 
offers a photograph was taken. These photographs together with the two offers formed the stimulus material used for the role of the proposers.

Offers significant for the present study resulted from 81 subjects (40 males) and were either fair (1/3 of the total amount) to all three players, unfair to the responder and the third player (both received less than 15\%), or unfair to only one player (receiving less than 15\%), whereas one of the others received at least one third of the whole amount. In total each of these four different categories consisted of 27 offers. The remaining 54 offers were neither really unfair (less than 15\%) nor fair (1/3 of the total amount). In all these conditions the proposers allocated at least $1 / 3$ of the total amount to themselves. In total 162 offers were presented in six blocks with containing 27 offers each.

\section{PARADIGM AND PROCEDURE}

Participants played as responders in a modified version of the three-person ultimatum game (Güth and van Damme, 1998). To ensure that participants believed in the presence of a dummy player, i.e., a third player, subjects were informed as part of the cover story that a second subject of the same gender as the subject him/herself participated in the experiment in a different EEG lab within the same building. In order to increase the feasibility of this setup, subjects were introduced to a second experimenter who supposedly was in charge of preparing the third player for EEG recordings and for running the experiment in the other lab.

All subjects received written instructions about the experimental task and were informed that they themselves, as well as the other players, would receive the amount of money from four randomly chosen trials. To save money only the four most successful proposers would receive compensation. Furthermore, subjects were shown the questionnaires filled out by the proposers to emphasize that the proposals were made by real persons. To avoid possible effects on the decisions to be made due to the physical appearance of the proposers, photographs were not presented prior to each offer (cf. Solnick and Schweitzer, 1999), butfollowing a suggestion by Knoch and colleagues (2006) — prior to each of the six blocks.

Hence, each block started with the presentation of photographs of the 27 proposers of the upcoming trials, followed by 27 offers which subjects had to accept or reject (Figure 1). The presentation of these offers, written in German (light gray background, black font color), consisted of three lines: the first line always contained the amount the proposer wanted to keep (e.g., "John gets $4 €$ "), the second indicated the amount the responder, i.e., the participant, would receive (e.g., "You get $4 €$ "), and the third line indicated the amount the third player would get (e.g., "Player 2 gets $4 €$ "). After $4000 \mathrm{~ms}$ two squares appeared below the offer, each of which either contained the word "accept" or "reject." These two alternatives changed the position randomly among the trials. Subjects were instructed to press the corresponding button of a response pad (PST Serial Response Box by Psychology Software Tools, Inc.) with their right hand to indicate the chosen alternative. Immediately after the response, a feedback of the actual allocation (format similar to the offers) was given for $2000 \mathrm{~ms}$. A variable interval of a mean duration of $2500 \mathrm{~ms} \pm 200 \mathrm{~ms}$ presenting a black fixation cross on a light gray screen separated the trials (offers). At the end of each block subjects were informed about the amount of money they had gained so far. To further ensure that participants believed in the presence of the other participant, i.e., the third player, 12 randomly chosen trials were followed by questions concerning the current offer (e.g., "Was the proposer male or female?"). Subjects were told that these questions have to be answered by the third player to maintain his or her attention to the task. As there was no real dummy player, answers in reality were given by the experimenter who was in a different room. Initiated at the subjects' own pace,

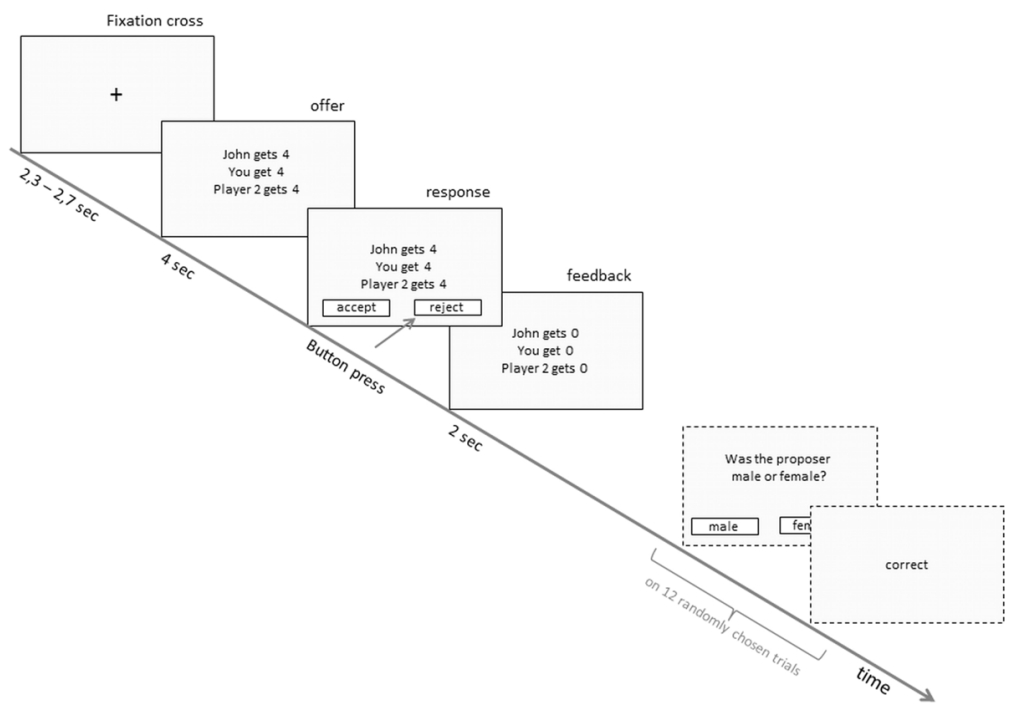

FIGURE 1 | Single-trial setting. Trials started with a fixation cross with a variable time interval, followed by the presentation of the offer. After the duration of $4 \mathrm{~s}$ two boxes appeared at the bottom of the screen indicating that participants can respond. Upon pressing the button, the feedback was presented. On 12 randomly chosen trials the feedback was followed by questions to be answered by the third player. 
the next block of trials started, again with the introduction of the subsequent proposers.

Stimulus presentation was controlled by a Pentium IV 3.00 GHz computer and E-prime software (E-prime 2.0, Psychology Software Tools, Inc., Pittsburgh, Pennsylvania). The whole experiment lasted for approximately $50 \mathrm{~min}$ including the short breaks between the blocks. After the experimental session the subjects were debriefed, i.e., they were informed about the purpose of the experiment and the fact that no real third player had been present.

\section{ELECTROPHYSIOLOGICAL RECORDINGS}

Participants were seated comfortably in a sound-attenuated and dimly lit room in front of a 19 inch cathode ray tube monitor. EEG data from each subject were recorded via $61 \mathrm{Ag} / \mathrm{AgCl}$ equidistantly located scalp electrodes embedded in an elastic cap (EASYCAP GmbH, Herrsching, Germany; montage M10), referenced to non-cephalic balanced sterno-vertebral electrodes (Stephenson and Gibbs, 1951). For eye movement artifact correction vertical and horizontal electro-oculograms (VEOG, HEOG) were recorded bipolar from above and below the left eye VEOG and from right and left outer canthi HEOG. The subjects' skin was slightly scratched with a sterile needle at all recording sites in order to minimize skin potential artifacts and to ascertain homogeneous electrode impedances below $2 \mathrm{k} \Omega$. Signals were amplified using a DC-amplifier with high baseline stability and an input impedance of $100 \mathrm{G} \Omega$ (Ing. Kurt Zickler GmbH, Pfaffstätten, Austria). Signals were digitized with a $1 \mathrm{kHz}$ sampling rate and recorded within a frequency range from DC to $250 \mathrm{~Hz}$.

\section{DATA PROCESSING AND ANALYSIS}

Reaction times (RT) were transformed using a logarithmic function (Knutson et al., 2007). RTs were then analyzed by means of a repeated-measures ANOVA with the two within-subjects factors self-related fairness (levels: fair, unfair) and other-related fairness (levels: fair, unfair); the first being the assignment to the responder and the second to the dummy player. For the comparison of rejection rates, a Friedman test was used.
Eye movement and blink artifacts were first eliminated using a linear regression approach on the basis of parameters obtained in pre-experimental calibration trials (Bauer and Lauber, 1979). Blink coefficients were identified using a template matching procedure. Blink correction was then performed by subtracting vertical and horizontal EOG signals weighted this way from each EEG channel. Subsequently, epochs of $800 \mathrm{~ms}$ following the presentation onset of the offer were extracted and baseline-corrected by subtracting the mean amplitude in the interval $200 \mathrm{~ms}$ before presentation onset. Data were then down-sampled to $250 \mathrm{smp} / \mathrm{s}$ and low pass filtered (6dB/octave slope) at $30 \mathrm{~Hz}$ cutoff. Before averaging the data were detrended, i.e., linear trends in the EEG signals were removed using the function "detrend" provided by EEGLAB 6.03b (Delorme and Makeig, 2004). To further improve data quality, e.g., correcting for residual artifacts occurring repeatedly, we followed the approach as outlined in Delorme et al. (2007): trials containing strong non-stereotype artifacts like movement or muscle-artifacts were rejected from further analysis based on visual inspection followed by an independent component analysis (ICA) using the extended infomax algorithm (Bell and Sejnowski, 1995; Lee et al., 1999) as implemented in the EEGLAB toolbox 6.03b (Delorme and Makeig, 2004). Individual independent components were screened for time courses and maps reflecting typical artifacts and then removed by back-projecting only the remaining, non-artifact components to the voltage time series.

Based on visual inspection of the grand-averaged waveforms and scalp distributions of difference waves (Figures 2 and $\mathbf{3}$ ) the MFN was quantified as the average baseline-corrected amplitude value in the time range between 240 and $340 \mathrm{~ms}$ after stimulus (offer) onset at electrode FCz (Boksem and de Cremer, 2010; Wu et al., 2011). To reduce confounding effects of other ERP components on the amplitude of the MFN, we created difference waves by subtracting ERPs elicited by offers with an equal share for all three players from the ERPs elicited by each of the three inequitable offers (unfair share for the subject, the dummy, or both). Additionally we created two difference waves by subtracting MFNs during Other fair from Other unfair for the two levels of fairness for the subject (Self fair, Self unfair).
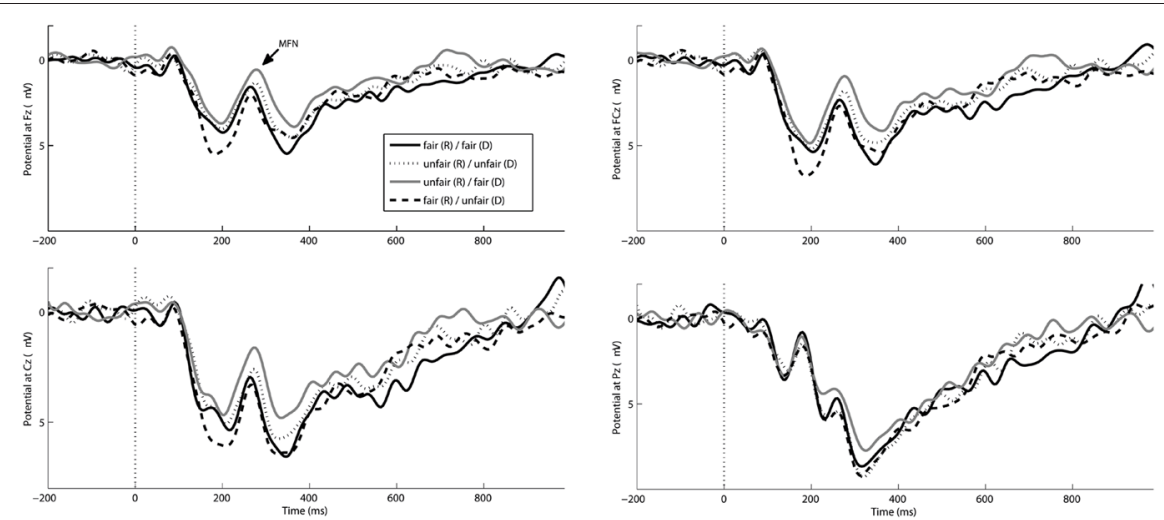

FIGURE 2 | Grand average ERP waveforms at Fz, Fcz, Cz, and Pz for the offers: fair/fair (solid line), fair/unfair (dashed line), unfair/fair (gray line), or unfair/unfair (dotted line). Negative is plotted up, Zeros on the timeline indicate the onset of the offer; format: responder/dummy. 


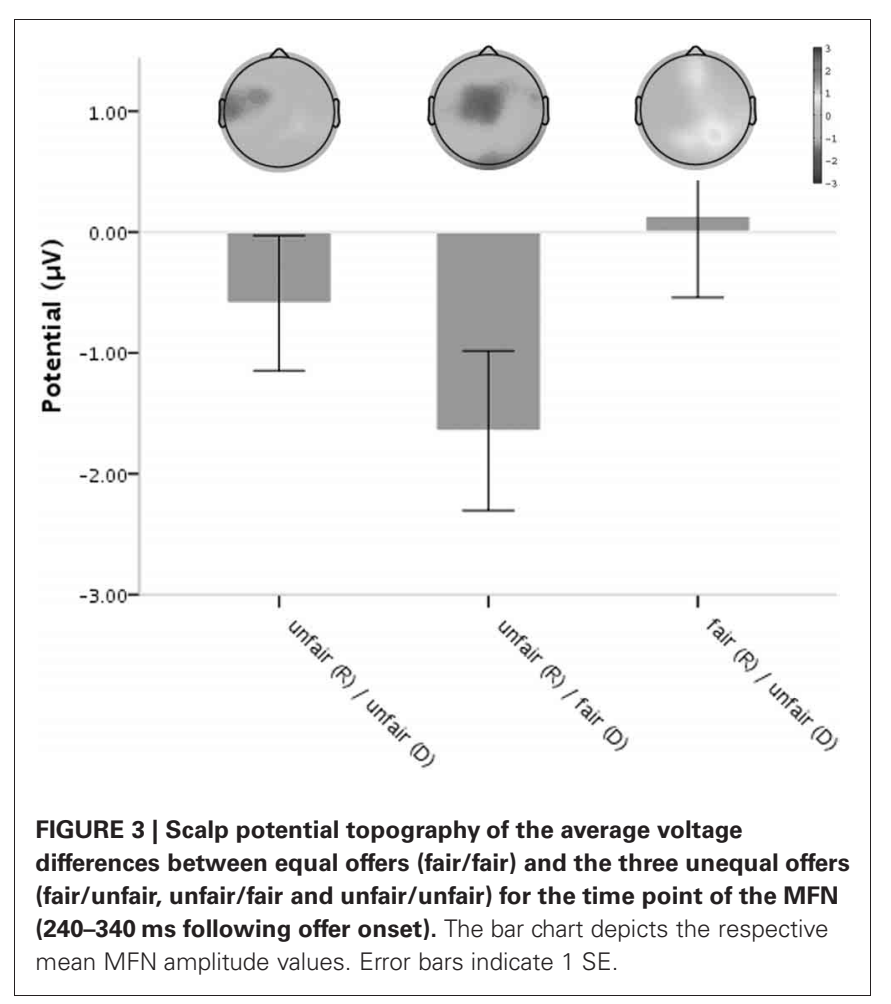

MFN amplitudes of difference waves were quantified as the average voltage in the $280-360 \mathrm{~ms}$ time interval at $\mathrm{FCz}$, against the pre-stimulus baseline.

MFN amplitude values at the selected location were submitted to separate $2 \times 2$ repeated measurement ANOVAs with the factors Self (levels: fair and unfair offers to the responder) and Other (levels: fair and unfair offers to the dummy player). These analyzes were aimed to describe whether an observed effect can be interpreted in terms of the offer made to the responder (factor Self), or to the dummy player (factor Other; Boksem et al., 2011). All factors were defined as within-subject factors. The degrees of freedom for repeated measures ANOVAs were Greenhouse-Geisser corrected whenever appropriate. To test whether difference waves are significantly different from zero a one-sample $t$-test was applied to the average voltage between 280 and $360 \mathrm{~ms}$ at FCz. In addition, to scrutinize potential differences in processing the outcome for the powerless third, controlled for the two outcomes for the responding subject, we conducted a paired sample $t$-test on MFN difference waves. For all analyses the significance threshold was set to $p \leq 0.05$. Finally, to assess the relation between early neuronal processes and actual behavior, MFN amplitudes for each condition as well as the associated difference waves (unfair minus fair) at channel FCz were analyzed in relation to the rejection rates of unequal offers using Pearson correlation coefficients (using directed, one-tailed significance levels; based on the results of Hewig et al., 2011).

\section{RESULTS}

\section{BEHAVIORAL RESULTS}

Analysis of the RTs (see Table 1) revealed a significant main effect for the factor Self $\left[F(1,15)=9.591, p=0.031, \eta^{2}=0.275\right]$
Table 1 | Behavioral results from the three-person ultimatum game.

\begin{tabular}{|c|c|c|c|c|}
\hline & fair $^{R} /$ fair $^{D}$ & fair $^{R} /$ unfair $^{D}$ & unfair $R /$ fair $^{D}$ & $\begin{array}{l}\text { unfair }^{R} / \\
\text { unfair }^{D}\end{array}$ \\
\hline Mean RT & 1076.32 & 1221.96 & 1230.31 & 1150.16 \\
\hline (SD) & (227.62) & (251.42) & (220.62) & (231.59) \\
\hline $\begin{array}{l}\text { Rejection } \\
\text { Rate }\end{array}$ & $3.00 \%$ & $39.35 \%$ & $64.96 \%$ & $70.37 \%$ \\
\hline
\end{tabular}

Reaction times (in $\mathrm{ms}$ ) with standard deviations in brackets and relative frequencies of rejections (in \%) are given.

and a significant Self $\times$ Other interaction $[F(1,15)=5.682, p=$ 0.007, partial $\left.\eta^{2}=0.390\right]$. In case both-responder and dummy-received an equally high share the shortest mean RTs (1076.85 ms, SD = 227.62) were observed, while offers unfair to the responder but fair to the dummy showed the longest mean RT $(1230.31 \mathrm{~ms}, \mathrm{SD}=220.62)$. There was a statistically significant difference in rejection rates depending on the type of offer, $\chi^{2}(3)=22.552, p<0.001$. Post-hoc analyzes with Wilcoxon Signed-Rank Tests were conducted with a Bonferroni correction applied, resulting in an individual significance level of $P=0.008$. There was no significant difference in rejection rates when comparing offers with an unfair share for the responder (unfair/fair vs. unfair/unfair; $Z=-1.398, p=0.162$ ). Despite an overall reduction in rejection rates for offers with a comparatively low share for the dummy (fair/unfair), rejection rates did not significantly differ from offers with a low share for both players (unfair/unfair; $Z=-2.120, p=0.034$ ) or offers with a low share for the responder (unfair/fair, $Z=-2.552, p=0.011$ ). However, when both players received a fair share offers were accepted significantly more often than all the other possible offers (all $p<0.001)$.

\section{ERP DATA}

Analysis of the MFN amplitude revealed a significant main effect for Self, $\left[F(1,15)=5.589, p=0.032\right.$, partial $\left.\eta^{2}=0.271\right]$ whereas, the factor Other did not reach significance $[F(1,15)=$ 1.033, $p>0.10$, partial $\left.\eta^{2}=0.064\right]$. There were no significant interaction effects $\left[F(1,15)=1.253, p>0.10\right.$, partial $\eta^{2}=$ 0.077]. The largest, more negative going, MFN amplitude was found for offers where only the responder received a low share (mean $\pm \mathrm{SD}, 1.79 \mu \mathrm{V} \pm 3.64$ ), and offers assigning a low share to only the dummy were accompanied by the least pronounced MFN amplitude $(3.56 \mu \mathrm{V} \pm 2.28$; see Figure 2). Thus, the MFN only distinguishes between fair and unfair offers for the responder, being larger for unfair offers, irrespective of the share for the dummy. Analysis of difference waves confirmed this finding. Again, the amplitude of the difference wave associated with offers that comprise a low share for the responder and a high share for the other players (unfair/fair minus fair/fair) was most pronounced and significantly different from zero (mean $=-1.643$, $t(15)=-2.491, p=0.025, d=0.881)$. Otherwise, subtracting ERPs elicited by equal offers (fair/fair) from ERPs elicited by offers with a low share for the dummy (fair/unfair) did not yield a difference wave significantly different from zero $[t(15)=0.199, p=$ $0.845, d=0.069]$. In addition, comparing unfair offers assigned 
to the dummy when responders themselves received a fair share as compared to an unfair share did not yield a significant difference either $\left[t(15)=1.119, p=0.281, d_{z}=0.028\right]$. The amplitudes of the difference waves reached their maximum $310 \mathrm{~ms}$ following offer onset, with a scalp distribution peaking over the fronto-central area (Figure 3). The same applied for P3 neither main effects nor interactions reached the level of significance (all $p>0.093$ ).

To investigate the relationship between electrophysiological data and behavioral choices we conducted correlation analyzes. Previous research on the standard ultimatum game found that MFN amplitudes following fair offers were related to rejection rates of offers with unequal splits (Hewig et al., 2011). Even though this statistical relationship could not be explained by their data and had not been measured or reported in previous studies, similar results were obtained in the present study: MFN amplitudes associated with equal offers were related to rejection rates of offers with an unequal split (see Table 2). Notably, this was only the case when responders themselves received a low share: rejections rates of offers with low shares for both players, the responder and the dummy player, were positively related to the MFN amplitude associated with equally fair offers $(r=0.46, p=0.037)$. Likewise, responders who frequently rejected offers with a low share for themselves exhibited smaller MFN amplitudes following equal offers $(r=0.60, p=0.007)$. No correlations $(p>0.475)$ were observed for the other correlation analyses (see Table 2 for all correlations).

\section{DISCUSSION}

The aim of this study was to investigate how the behavioral and neural responses of a responder in an ultimatum game are affected by a third, passive player. Since the MFN has been associated with early evaluation processes in economic decisionmaking, the analysis focused on this ERP component. Usually the $\mathrm{MFN}$ is more negative going when people experience an undesirable outcome or event compared to a more acceptable one. We assumed that involving a third player might affect the way people evaluate their payoffs, and in turn modulate the MFN amplitudes. To this end, we recorded EEG from participants playing in the role of the responders in a modified version of the three-person ultimatum game. Overall, the results indicate that people dissociate between high and low offers assigned to them. In particular, the amplitude differences about $300 \mathrm{~ms}$ after the presentation of the offer can be mainly explained by the share for oneself, while the non-significant main effect for other-related fairness suggests that the subjects' neural responses were not indexing the

Table 2 | Correlations between mean MFN amplitudes for equal (fair/fair) offers and rejection rates of the different offers ${ }^{*} p<0.01$, ${ }^{* *} p<0.05$.

\begin{tabular}{cclll}
\hline & fair $^{R} /$ fair $^{D}$ & fair $^{R} /$ unfair $^{D}$ & unfair $^{R} /$ fair $^{D}$ & $\begin{array}{l}\text { unfair }^{R} / \\
\text { unfair }^{D}\end{array}$ \\
\hline $\begin{array}{c}\text { MFN at } \\
\text { FCz }\end{array}$ & -0.071 & 0.017 & $0.598^{* *}$ & $0.458^{* *}$ \\
\hline
\end{tabular}

fairness of offers to the powerless third. In addition, no effect was found when comparing difference waves between fair and unfair offers assigned to the powerless third. Furthermore, though there seems to be a relation between MFN amplitude and behavior in the ultimatum game (Hewig et al., 2011), this effect was only observed with offers that negatively affected the responder's payoff.

Previous ERP studies on the two-person ultimatum game have shown that offers with a low share for the responder were associated with more pronounced MFN amplitudes as compared to offers with equal ones (Boksem and de Cremer, 2010; Hewig et al., 2011; Wu et al., 2011). The MFN is believed to reflect a subjective motivational judgment indicating whether an event or outcome is better or worse than expected (Gehring and Willoughby, 2002; Nieuwenhuis et al., 2004). In line with this assumption people who are more concerned about fairness norms exhibit more pronounced MFN amplitudes following norm violations in the ultimatum game (Boksem and de Cremer, 2010). Furthermore, several authors have shown that an MFN can be observed when gambling task outcomes refer to someone else (van Schie et al., 2004; Fukushima and Hiraki, 2006). Even when the task performance of others does not affect the subjects themselves, particularly those with high trait empathy have higher MFN amplitudes when the other person makes a mistake and loses money (Fukushima and Hiraki, 2009). Therefore, we assumed that the MFN would be as well modulated by an unfair share toward the third player. According to a recent neuroimaging study advantageous inequity as compared to disadvantageous inequity is less rewarding as indicated by reduced activity in brain areas that are associated with reward processing. Subjects who were better off in material terms than their counterparts showed less activity in ventral striatum and ventromedial prefrontal cortex when they received money that augmented the difference in payoff (Tricomi et al., 2010). Following the concept of inequality aversion, we expected unequal offers always to be associated with higher MFN amplitudes than equal ones. This assumption was not confirmed by the data. A recent study using the two-person ultimatum game found that MFN amplitude differences following fair and unfair offers were not modulated by observing the allocation outcome of other unrelated responder-proposer dyads (Wu et al., 2011). Involving a third player we found similar results as $\mathrm{Wu}$ and colleagues, suggesting that neither an external reference point nor a fixed reference agent clearly modulate this early ERP component. Furthermore, in a social comparison task prosocial subjects responded with higher amygdala activity and felt more unpleasant in response to unequal payoffs. Individualist showed the opposite pattern, i.e., an increase in reward difference was associated with decreased activity in the amygdala (Haruno and Frith, 2010). The ACC, the dorsolateral prefrontal cortex and the anterior insula, i.e., brain areas that have been associated with the processing of unfair offers in the ultimatum game (Sanfey et al., 2003), were found to show higher activity when subjects received less than their counterpart. Interestingly those regions were similarly activated in prosocial subjects and individualist. Since it is supposed that the MFN is generated in the ACC according to Gehring and Willoughby (2002), our results broaden these findings due to the 
higher temporal resolution of the EEG and suggest that the initial response is mainly self-related. Nevertheless, it is important to remember that the three players in our experiment remained anonymous to each other, which is the standard procedure in these kind of paradigms (Camerer and Richard, 1995; Güth et al., 2007). This is in contrast to studies that found evidence for a component that might be interpreted as an other-related MFN where pairs were either seated in the same room or knew each other already prior to the experiment (Fukushima and Hiraki, 2006, 2009). Thus, it might be possible that decreasing the degree of psychological or physical distance between players might have resulted in different behavioral and neuronal responses.

However, concerning the behavioral responses we found consistency with previous behavioral studies. Participants preferred equal shares, whereas offers with a low share for the responder were rejected most frequently (Güth et al., 2007). Furthermore, in line with previous ERP studies (Boksem and de Cremer, 2010; Hewig et al., 2011) no relation between the amplitude of the MFN difference wave and rejection rates was found. Nevertheless, the MFN amplitude following equitable offers was highly related to rejection rates of offers with low shares for the responder. This finding has already been reported in previous studies on the two-person ultimatum game (e.g., Hewig et al., 2011). Several studies on the MFN are based on the assumption that alterations of the amplitudes are solely related to the processing of negative events or events that are worse than expected (Gehring and Willoughby, 2002; Nieuwenhuis et al., 2004). According to Holroyd and Coles (2002, 2008), though, unexpected negative events would have an enhancing effect, unexpected positive events would have an attenuating effect on the MFN amplitude. Pedroni and colleagues (2011) argue that there are actually two processes that occur at the time a MFN can be observed, an evaluation on a good-bad dimension, as proposed in previous studies, and the evaluation of the (positive) reward value. In light of the assumption that positive and particularly unexpected positive events lead to a change in MFN amplitude, Hewig and colleagues (2011) propose two possible explanations for the relation between rejections rates and MFN amplitudes following equal offers. Either more reward sensitive participants reject unfair offers because they are disappointed, or those who expect others to be selfish are more likely to reject unfair offers, due to their negative view of others. More precisely, if offers with a high share for oneself are related to stronger reward-related responses, these offers are also accompanied by a reduction in MFN amplitude. In this regard participants with lower MFN amplitudes following equal offers are more disappointed when offered a relatively small amount and hereupon reject these proposals. On the other hand, if participants believe that proposers are rather selfish by keeping most of the money for themselves, they may expect receiving mainly unequal offers. This negative view of others might lead to higher rejection rates and smaller MFN amplitudes in relation to equal "better than expected" proposals. Our findings provide some new insights on this relation between neural and behavioral response. In the study by Hewig and colleagues (2011) offers with an equal amount for both players represented the highest possible reward for the responder. In the present study there are two possible conditions with high shares for the responder. Equal offers as well as advantageous unequal offers denote a high share for the responder. Assuming that reward sensitivity predicts decision behavior, both offers with high shares for the responder should be related to rejection rates or at least should be related to each other. Yet, such a relation could not be established in the present study. Only equal offers were related to decision behavior. This evidence suggests that in the context of social interaction our expectations concerning the behavior of others might already guide our own behavior. Similarly, in contradiction to the hypothesis that negative emotions following unfair offers might facilitate memory for cheaters (Mealey et al., 1996; Vanneste et al., 2007; Barclay, 2008), a recent study on the standard two-person ultimatum game found that the proposers' behavior per se does not enhance memory. Conversely, when offers did not meet the expectations of the responders, they remembered the proposers' face more efficiently (Chang and Sanfey, 2009).

Importantly, the so-called power coalition-the third player receives far less than the other two players-was rejected quite frequently. Rejections of those offers were not at all related to MFN amplitudes. Similarly, a recent study measuring skin conductance response (SCR) revealed that an inequitable offer in the standard two-person game is followed by an increase in SCR. However, when people are playing the two-person ultimatum game on behalf of another person, they do not show this increase in SCR following an unfair offer. Yet, these offers were rejected as often as when playing for themselves. Therefore, affective responses were solely related to self-relatedness, while behavioral responses were not (Civai et al., 2010). This suggests that economic decisions are not necessarily always related to the emotional response-in particular when there is enough time for a controlled, deliberative process. Yet, when these deliberative processes are inhibited by time pressure, decisions as in the ultimatum game are only guided by affective processes as indicated by an increase in rejection rate (Sutter et al., 2003; Cappelletti et al., 2008). In the present study participants had unlimited time to decide whether to accept or reject an offer, which might also explain the discrepancy between the early neuronal and the subsequent behavioral response, especially with regard to offers that affect the third, passive player. Nevertheless, note that the neuroimaging study on inequality aversion by Haruno and Frith (2010) suggests that the amygdala activity in response to unequal reward pairs reflects a rapid intuitive response. This assumption is based on the finding that cognitive load had no effect on inequality aversion. Evaluation of reward differences and RT did not differ in a high cognitive load compared to a low-load condition. This is in line with behavioral studies on the ultimatum game that found no difference in rejection rates under cognitive load, whereas, responders reject unfair offers more often under time pressure (Sutter et al., 2003; Cappelletti et al., 2008). Of course, it would have been interesting to elucidate later parts of the decision making process, but this is beyond the scope of the present work. Nevertheless, the relation between the different parts of the decision-making process is of central importance to gain a more accurate and exhaustive understanding of (economic) decision-making. 
Taken together, the results of the present study show that inequality aversion cannot explain variability in the early neuronal evaluation process. On an early neuronal level, humans dislike disadvantageous inequality and seem to favor advantageous inequality. Although the decision behavior observed in this study suggests that humans care about the powerless third, there is no evidence for an early affective response suggesting that subjects do not care about what the other person receives. Thus, we propose that the first automatic response to inequality

\section{REFERENCES}

Barclay, P. (2008). Enhanced recognition of defectors depends on their rarity. Cognition 107, 817-828.

Bauer, H., and Lauber, W. (1979). Operant conditioning of brain steady potential shifts in man. Biofeedback Self Regul. 4, 145-154.

Bell, A. J., and Sejnowski, T. J. (1995). An information-maximization approach to blind separation and blind deconvolution. Neural Comput. 7, 1129-1159.

Boksem, M. A., and de Cremer, D. (2010). Fairness concerns predict medial frontal negativity amplitude in ultimatum bargaining. Soc. Neurosci. 5, 118-128.

Boksem, M. A., Kostermans, E., and de Cremer, D. (2011). Failing where others have succeeded: medial frontal negativity tracks failure in a social context. Psychophysiology 48, 973-979.

Bolton, G. E., and Ockenfels, A. (2000). ERC: a theory of equity, reciprocity, and competition. Am. Econ. Rev. 90, 166-193.

Camerer, C. T., and Richard, H. (1995). Anomalies: ultimatums, dictators and manners. J. Econ. Perspect. 9, 209-219.

Cappelletti, D., Güth, W., and Ploner, M. (2008). Being of two minds: an ultimatum experiment investigating affective processess. Jena Econ. Res. Pap. 2008, 048.

Chang, L. J., and Sanfey, A. G. (2009). Unforgettable ultimatums? Expectation violations promote enhanced social memory following economic bargaining. Front. Behav. Neurosci. 3:36. doi: 10.3389/neuro.08.036.2009

Civai, C., Corradi-Dell'Acqua, C., Gamer, M., and Rumiati, R. I. (2010). Are irrational reactions to unfairness truly emotionallydriven? Dissociated behavioural and emotional responses in the ultimatum game task. Cognition 114, 89-95.

Delorme, A., and Makeig, S. (2004). EEGLAB: an open source toolbox for analysis of single-trial EEG dynamics including independent component analysis. J. Neurosci. Methods 134, 9-21.

Delorme, A., Sejnowski, T., and Makeig, S. (2007). Enhanced detection of artifacts in EEG data using higher-order statistics and independent component analysis. Neuroimage 34, 1443-1449.

Fehr, E., and Schmidt, K. M. (1999). A theory of fairness, competition, and cooperation. Q. J. Econ. 114, 817-868.

Fukushima, H., and Hiraki, K. (2006). Perceiving an opponent's loss: gender-related differences in the medial-frontal negativity. Soc. Cogn. Affect. Neurosci. 1, 149-157.

Fukushima, H., and Hiraki, K. (2009). Whose loss is it? Human electrophysiological correlates of non-self reward processing. Soc. Neurosci. 4, 261-275.

Gehring, W. J., and Willoughby, A. R. (2002). The medial frontal cortex and the rapid processing of monetary gains and losses. Science 295, 2279-2282.

Guroglu, B., van Den Bos, W., van Dijk, E., Rombouts, S. A., and Crone, E. A. (2011). Dissociable brain networks involved in development of fairness considerations: understanding intentionality behind unfairness. Neuroimage 57, 634-641.

Güth, W., Schmidt, C., and Sutter, M. (2007). Bargaining outside the lab a newspaper experiment of a threeperson ultimatum game. Econ. J. 117, 449-469.

Güth, W., Schmittberger, R., and Schwarze, B. (1982). An experimental analysis of ultimatum bargaining. J. Econ. Behav. Organ. 3, 367-388.

Güth, W., and van Damme, E. (1998). Information, strategic behavior, and fairness in ultimatum bargaining: an experimental study. J. Math. Psychol. 42, 227-247.

Haruno, M., and Frith, C. D. (2010). Activity in the amygdala elicited by unfair divisions predicts social value orientation. Nat. Neurosci. 13, 160-161.

Hewig, J., Kretschmer, N., Trippe, R. H., Hecht, H., Coles, M. G.,

is mainly self-related, whereas, concerns for the well-being of others are part of higher cognitive, deliberative or intuitive processes following the first automatic response.

\section{ACKNOWLEDGMENTS}

Johanna Alexopoulos is a recipient of a DOC-fFORTE-fellowship of the Austrian Academy of Science. This research has partially been supported by the Austrian Science Fund (FWF): P22813-B09 (to Claus Lamm).

Holroyd, C. B., and Miltner, W. H. (2011). Why humans deviate from rational choice. Psychophysiology 48, 507-514.

Holroyd, C. B., and Coles, M. G. (2002). The neural basis of human error processing: reinforcement learning, dopamine, and the errorrelated negativity. Psychol. Rev. 109, 679-709.

Holroyd, C. B., and Coles, M. G. (2008). Dorsal anterior cingulate cortex integrates reinforcement history to guide voluntary behavior Cortex 44, 548-559.

Knoch, D., Pascual-Leone, A., Meyer K., Treyer, V., and Fehr, E. (2006). Diminishing reciprocal fairness by disrupting the right prefrontal cortex. Science 314, 829-832.

Knutson, B., Rick, S., Wimmer, G. E., Prelec, D., and Loewenstein, G. (2007). Neural predictors of purchases. Neuron 53, 147-156.

Lee, T.-W., Girolami, M., and Sejnowski, T. J. (1999). Independent component analysis using an extended infomax algorithm for mixed subgaussian and supergaussian sources. Neural Comput. 11, 417-441.

Martin, L. E., Potts, G. F., Burton, P. C., and Montague, P. R. (2009). Electrophysiological and hemodynamic responses to reward prediction violation. Neuroreport 20, 1140-1143.

Mealey, L., Daood, C., and Krage, M. (1996). Enhanced memory for faces of cheaters. Ethol. Sociobiol. 17, 119-128.

Miltner, W. H. R., Braun, C. H., and Coles, M. G. H. (1997). Eventrelated brain potentials following incorrect feedback in a timeestimation task: evidence for a "Generic" neural system for error detection. J. Cogn. Neurosci. 9, 788-798.

Nieuwenhuis, S., Holroyd, C. B., Mol, N., and Coles, M. G. (2004) Reinforcement-related brain potentials from medial frontal cortex: origins and functional significance. Neurosci. Biobehav. Rev. 28, 441-448.
Oldfield, R. C. (1971). The assessment and analysis of handedness: the Edinburgh inventory. Neuropsychologia 9, 97-113.

Pedroni, A., Langer, N., Koenig, T., Allemand, M., and Jäncke, L. (2011). Electroencephalographic topography measures of experienced utility. J. Neurosci. 31, 10474-10480.

Polezzi, D., Daum, I., Rubaltelli, E., Lotto, L., Civai, C., Sartori, G., and Rumiati, R. (2008). Mentalizing in economic decision-making. Behav. Brain Res. 190, 218-223.

Rilling, J. K., and Sanfey, A. G. (2011). The neuroscience of social decisionmaking. Annu. Rev. Psychol. 62, 23-48.

Sanfey, A. G., Rilling, J. K., Aronson, J. A., Nystrom, L. E., and Cohen, J. D. (2003). The neural basis of economic decision-making in the ultimatum game. Science 300, 1755-1758.

Schultz, W. (1999). The reward signal of midbrain dopamine neurons. News Physiol. Sci. 14, 249-255.

Schultz, W. (2010). Dopamine signals for reward value and risk: basic and recent data. Behav. Brain Funct. 6, 24 .

Selten, R. (1965). Die Strategiemethode zur Erforschung des eingeschränkt rationalen Verhaltens im Rahmen eines Oligopolexperimentes. Seminar für Mathemat. Wirtschaftsforschung u. Ökonometrie.

Solnick, S. J., and Schweitzer, M. E. (1999). The influence of physical attractiveness and gender on ultimatum game decisions. Organ. Behav. Hum. Decis. Process. 79, 199-215.

Stephenson, W. A., and Gibbs, F. A. (1951). A balanced noncephalic reference electrode. Electroencephalogr. Clin. Neurophysiol. 3, 237-240.

Sutter, M., Kocher, M., and Strauß, S. (2003). Bargaining under time pressure in an experimental ultimatum game. Econ. Lett. 81, 341-347.

Tricomi, E., Rangel, A., Camerer, C. F., and O/'Doherty, J. P. (2010). Neural evidence for inequality-averse social preferences. Nature 463, 1089-1091. 
van Schie, H. T., Mars, R. B., Coles, M. G., and Bekkering, H. (2004). Modulation of activity in medial frontal and motor cortices during error observation. Nat. Neurosci. 7, 549-554.

Vanneste, S., Verplaetse, J., van Hiel, A., and Braeckman, J. (2007). Attention bias toward noncooperative people. A dot probe classification study in cheating detection. Evol. Hum. Behav. 28, 272-276.
Wu, Y., Zhou, Y., van Dijk, E., Leliveld, M. C., and Zhou, X. (2011). Social comparison affects brain responses to fairness in asset division: an ERP study with the ultimatum game. Front. Hum. Neurosci. 5:131. doi: 10.3389/fnhum.2011.00131

Conflict of Interest Statement: The authors declare that this research project was conducted in the absence of any commercial or financial relationship that could be construed as a potential conflict of interest.

Received: 30 December 2011; accepted: 05 March 2012; published online: 28 March 2012

Citation: Alexopoulos J, Pfabigan DM, Lamm C, Bauer H and Fischmeister FPS (2012) Do we care about the powerless third? An ERP study of the three-person ultimatum game. Front. Hum. Neurosci. 6:59. doi: 10.3389/fnhum.2012.00059 Copyright (c) 2012 Alexopoulos, Pfabigan, Lamm, Bauer and Fischmeister. This is an open-access article distributed under the terms of the Creative Commons Attribution Non Commercial License, which permits non-commercial use, distribution, and reproduction in other forums, provided the original authors and source are credited. 